Communications in Physics, Vol. 14, No. 2 (2004), pp. 100-104

\title{
GENERATION OF SINGLE FREQUENCY LIGHT FROM AN OPTICAL FEEDBACK SPECIAL LASER MODULE
}

\author{
TRAN THI PHUC \\ Military Centre of Science, Engineering and Technology \\ VU VAN LUC \\ Vietnamese Academy of Science and Technology
}

\begin{abstract}
Generation of single frequency radiation from a semiconductor laser based on a special laser module (amplifying laser module) is presented. The multi-mode emission with the spectral bandwidth of $100 A^{0}$ is converted to single mode one with a narrow bandwidth of $2 A^{0}$ in the presence of external optical feedback. Wavelength tunability of the laser emission over the range of 4-10 $\mathrm{nm}$ is also shown. The results could be used in high speed optical communication, WDM and other applications.
\end{abstract}

\section{INTRODUCTION}

In optical communication systems, the single frequency laser sources are essential in order to enhance the rate and transfer distance. In addition, the wavelength division multiplexing (WDM) technology has been strongly expanding over the world in which channels are simultaneously transfered in a common optical fiber, different channels are separated by carried wavelengths. The number of channels coupled into a fiber is determined by the monochromaticity of light pulses. Therefore, the single frequency tunable laser diode is necessary for WDM to get desired wavelength with low expense.

Basing on a special laser module produced at the Lab of semiconductor lasers, we have successfully generated tunable single frequency pulses from an external cavity diode laser.

\section{SPECIAL MODULE LASER BASED ON InGaAsP/InP EMITTTED AT THE REGION OF $1310 \mathrm{~nm}$}

In conventional laser modules, one facet of the laser diode is coupled with monomode optics fiber through a micro-lens, the another facet is directed to a photodiode monitor. In our special module, both facets of the laser cavity are coupled with two fibers. The coupling is carefully processed in such a way as to obtain the highest and equal efficiency at both facets. In fact, it is very difficult to couple diode with fiber because of the difference between the section of active region (of the order of $0.1-0.3 \mu \mathrm{m}$ ) and diameter of optic fiber $(7-9 \mu \mathrm{m})$. The coupled end of the fiber is formed as a micro-lens and the another end is connected to FC standard connector. The laser diode is fixed on a base which is attached with a temperature sensor. The temperature sensor, in turn, is controlled by a Peltier cooler. We have achieved special laser modules with considerably high coupling efficiency $(\geq 40 \%)$ and nearly equal at both sides. Fig. 1 shows the internal structure (a) and exterior picture (b) of the special laser module (amplifying laser module), which emits at region of $1310 \mathrm{~nm}$. 


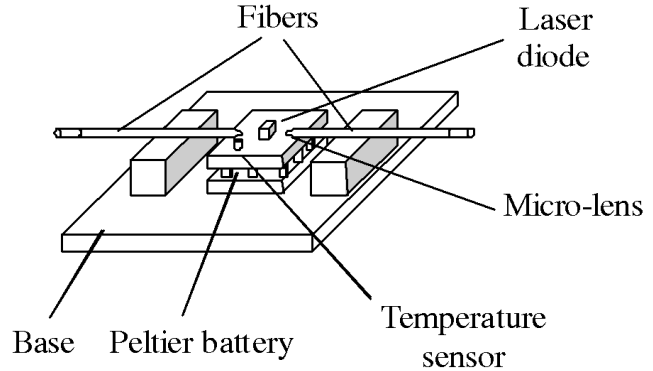

a)

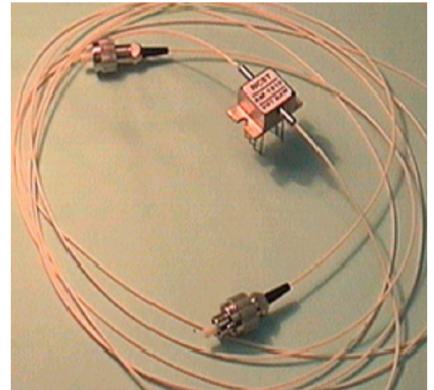

b)

Fig. 1. Special laser module: a - Internal structure, b - Exterior picture

\section{SPECIAL LASER MODULE WITH OPTICAL FEEDBACK}

Emission spectrum from a conventional diode laser has the form of multi-longtitude-mode. The side modes can be suppressed in CW operation but become significant in high repetition rate pulsed operation (spectral width could be double at high modulation rate). Particularly, in optical communication systems, laser diode is normally modulated at frequency of order of GHz. Due to the dispersion, undesired side modes lead to narrowing of fiber bandwidth which results in limitted transfer rate. Therefore, single frequency emission at high repetition rate from a diode laser has been expected. In normal laser diode with Fabry - Perot cavity, optical feedback is established by the from two cavity ends. In this case, the reflection is the same for longtitude modes. Obviously, in order to obtain single mode operation, spectrally dependent optical feedback must be set up in laser cavity so that cavity losses are different for longtitude modes. As a result, undesired side modes are suppressed. Practically, mechanism of distributed feedback and conjugated resonances are commonly used for that $[1,2]$. The latter one has been used in this paper. In fact, this is performed with a external cavity. The effect of this cavity on mode selection is outlined in Fig. 2.

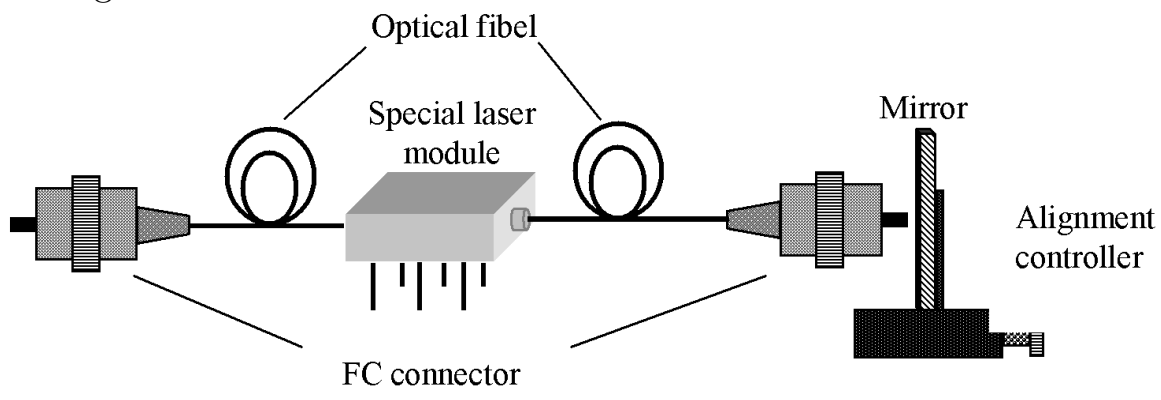

Fig. 2. External cavity of special laser module

Special laser modules are used with externally optical feedback from a separate mirror of $96 \%$ reflectance. One end of the connector faces to a mirror which is mounted on a positioner. Using piezoelectric transducer, the distance $d$ between the connector and mirror can be precisely controlled with accuracy of less than micrometers and allows us to conveniently monitor the effect of optical feedback while the distance changed. The another end of connector is used for studying the laser module characteristics. 
This structure provides an important advantage which is unique to special laser modules. It not only allows us to study with high accuracy but also to directly use in optical communication systems.

\section{SPECTRA OF SPECIAL LASER MODULES}
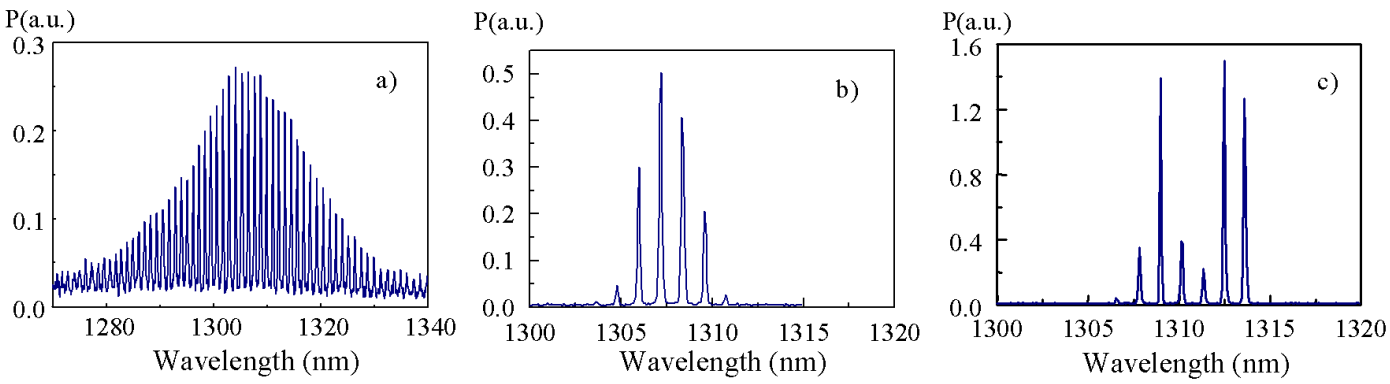

Fig. 3. Spectra of the special laser module No. $108\left(\mathrm{I}_{t h}=15 \mathrm{~mA}\right)$ at different operation currents $\mathrm{I}_{o p}$. a) $\mathrm{I}_{o p}=12 \mathrm{~mA}$; b) $\mathrm{I}_{o p}=15 \mathrm{~mA}$; c) $\mathrm{I}_{o p}=18 \mathrm{~mA}$

The special laser modules are based on InGaAsP/InP semiconductor laser diode with buried heterostructure. They emit at the region of $1310 \mathrm{~nm}$ with threshold currents $\left(\mathrm{I}_{t h}\right)$ of $15 \mathrm{~mA}$ (module $\mathrm{N}^{o} 108$ ) and of $13 \mathrm{~mA}$ (module $\mathrm{N}^{o} 109$ ). Emision spectra from laser module $\mathrm{N}^{\circ} 108$ with various currents are presented in Fig. 3.

As seen in Fig. 3, at pumping current of $12 \mathrm{~mA}$ (operation below threshold - Fig. 3a), superluminesce from the module covers a spectrally broad range from $1210 \mathrm{~nm}$ to $1340 \mathrm{~nm}$. With pumping currents greater than or equal threshold (Figs. 3b and 3c), laser emission spectra are constituted of $5-6$ axial modes. But with the increase in pumping current, distribution of mode intensities becomes irregular (Fig. 3c).

\section{INFLUENCES OF EXTERNALLY OPTICAL FEEDBACK ON CHARACTERISTICS OF SPECIAL LASER MODULE}

\section{V.1. Influences of feedback on laser thresh- old and power characteristics}

The dependence of output power on the operation current of the special laser module $\mathrm{N}^{o}$ 108 is shown in Fig. 4. The curve 1 corresponds to the operation without optical feedback. The threshold current is found to be $15 \mathrm{~mA}$ and output power at operation current of $20 \mathrm{~mA}$ is 0.48 $\mathrm{mW}$. In case of module operation with low feedback (connector - mirror distance of $100 \mu \mathrm{m}$ ) the threshold is reduced to $13 \mathrm{~mA}$ (curve 2). Besides that, output power at pumping current of $20 \mathrm{~mA}$ is risen to $0.75 \mathrm{~mW}$. With higher feedback (connector - mirror distance is reduced to $50 \mu \mathrm{m}$ ),

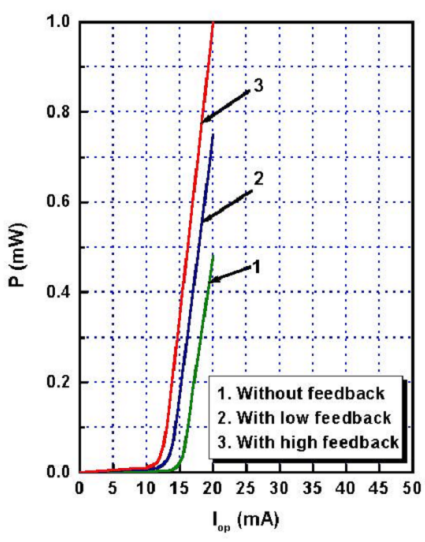

Fig. 4. Power characteristics without (1) and with feedback $(2,3)$ 
thershold decreased to $12 \mathrm{~mA}$ and output power reached $1 \mathrm{~mW}$ (curve 3). This is very useful for the practical use of the special laser module. It allows us to obtain highly sufficient output power at low operation current which avoids burning the module.

\section{V.2. Influences of feedback on spectral characteristics}

The experimental results of laser spectra at various operation currents are presented in Fig. 5. Evidently, initially broadband laser emission is narrowed to be monomode with the increase in current from $12 \mathrm{~mA}$ to $18 \mathrm{~mA}$.
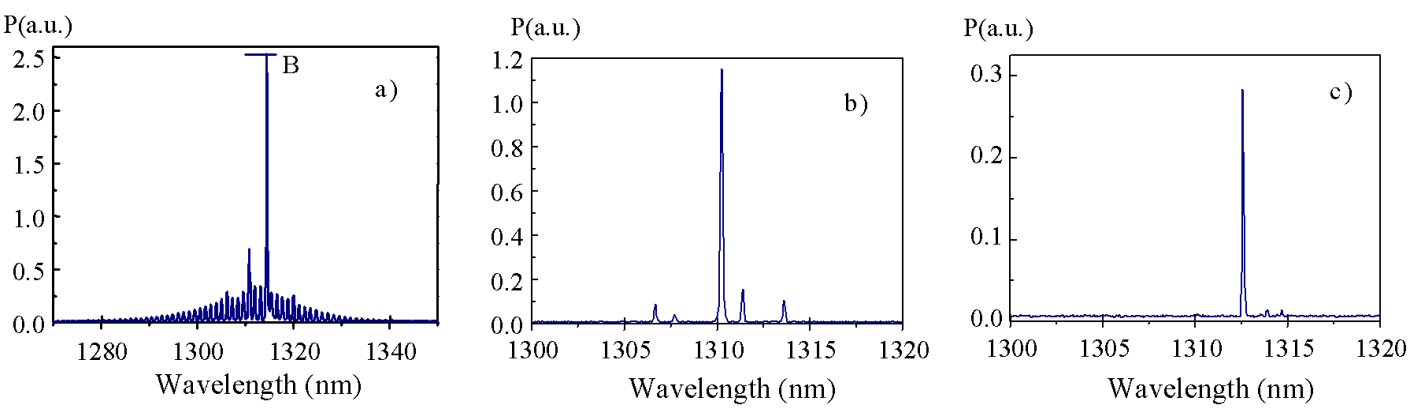

Fig. 5. Spectra of the laser module $\mathrm{N}^{o} 108$ with the feedback from a mirror $\mathrm{R}=96 \%$ at different operation currents. a) $\mathrm{I}_{o p}=12 \mathrm{~mA}$; b) $\mathrm{I}_{o p}=15 \mathrm{~mA}$; c) $\mathrm{I}_{o p}=18 \mathrm{~mA}$

Spectra of laser emission from module $\mathrm{N}^{o} 109$ without and with externally optical feedback are shown in Fig. 6 at operation current $\mathrm{I}_{o p}=\mathrm{I}_{t h}=13 \mathrm{~mA}$. In the presence of external feedback, laser spectrum is converted from multi-mode to monomode. Obviously, external feedback is a useful mechanism to generate monomode light pulses from laser modules.
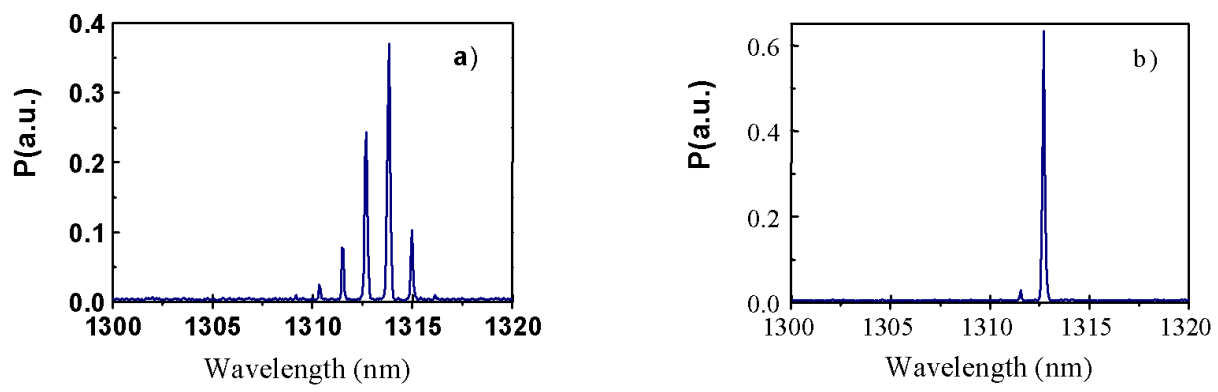

Fig. 6. Spectra of the special laser module $\mathrm{N}^{o}$. 109 without the feedback (a); and with the feedback from a mirror at distance of $50 \mu \mathrm{m}$ (b).

\section{MODE SHIFT IN WAVELENGTH}

Single frequency laser emission can be tuned by the change of refractive index of active region. Alternatively, the change in cavity length is possible to tune wavelength 
$[3,4]$. We have observed the mode shift in wavelength as voltage on piezoelectric transducer varied, i.e. d, the distance between FC connector and mirror, changed. (Fig. 7). This provides a convenient way to tune wavelength of laser emission.
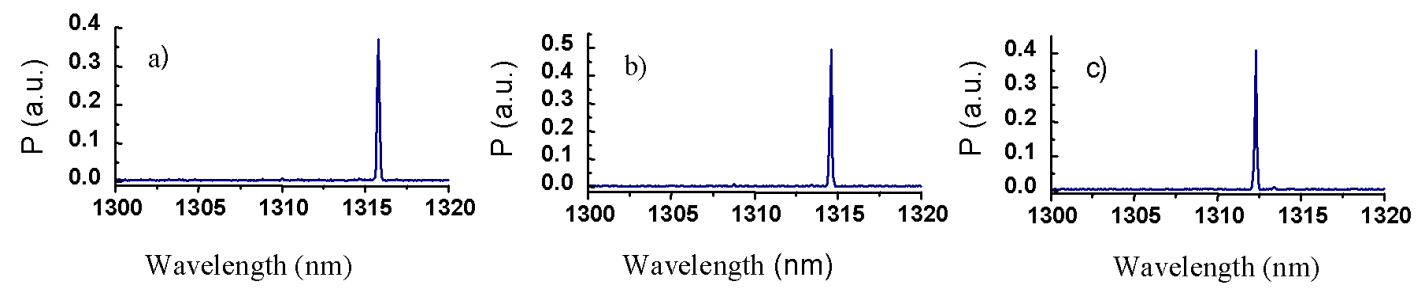

Fig. 7. Spectral shift of the No109 with feedback at operation current of $12 \mathrm{~mA}, \mathrm{~T}=$ $25{ }^{0} \mathrm{C}$ pzt $\left.=85 \mathrm{~V}(\mathrm{~d}=15,0 \mu \mathrm{m}) ; \mathrm{pzt}=90 \mathrm{~V}(\mathrm{~d}=14,5 \mu \mathrm{m}) ; \mathrm{c}\right)$ pzt $=95 \mathrm{~V}(\mathrm{~d}=14,0 \mu \mathrm{m})$.

\section{CONCLUSION}

The special laser modules has been successfully produced and used for studying externally optical feedback laser characteristics. The experimental results show the enhanced performance of special laser modules in comparision to conventional laser diode. The laser emission with optical feedback from a separate mirror is practically single frequency and can be tuned over the range of $5-10 \mathrm{~nm}$ by simply changing the gap between connector and mirror. In addition, use of external feedback provides some benefit including higher output and lower threshold current.

The obtained results are promising for application not only to high speed optical communication (600 Gbps/km), but also to spectrally high resolved spectroscopy [5].

\section{ACKNOWLEDGEMENT}

This investigation is carried out with the support from the Fundamental Research Program (KC-04). The authors would like to thank Ha Cong Nguyen, Vu Van Truong for their help.

\section{REFERENCES}

1. Preston K. R,K. Woolllard and K. H. Cameron, Electron. Lett., 17 (1981) 931.

2. R. Lang, IEEE J. Quantum Electron., 16 (1980) 347.

3. R. Wyatt, W. J. Devlin, Electron. Lett., 19 (1983) 110.

4. Yukata Ikuma, JunJi Ohtsubo, IEEE J. Quantum Electron., 34 (1998) 7.

5. D. Chu, R. Potenza, Laser Focus World, No. 8 (2001) 101. 